\title{
Sequenciamento de infusão de antineoplásicos: contribuições para a prática de enfermagem oncológica baseada em evidência
}

\author{
Angelo Braga Mendonça ${ }^{1}$, Eliane Ramos Pereira ${ }^{2}$, Carinne Magnago ${ }^{3}$, Bruna Maiara Ferreira Barreto ${ }^{4}$, \\ Ticiane Roberta Pinto Goes ${ }^{5}$, Rose Mary Costa Rosa Andrade Silva ${ }^{6}$
}

\section{RESUMO}

Este estudo objetivou identificar as evidências científicas disponíveis acerca das interações medicamentosas entre antineoplásicos decorrentes da ordem de infusão para, então, descrever a melhor sequência de aplicação e discutir sua aplicabilidade na Sistematização da Assistência de Enfermagem (SAE). Revisão integrativa da literatura operacionalizada em 2018 nas bases MEDLINE, LILACS, CINAHL e BVS, a partir dos termos Neoplasms, Drug Therapy, Drug Interactions, Chemotherapy e Sequence Administration. Foram analisados 57 estudos que, em conjunto, estudaram 40 combinações de antineoplásicos, apontando as interações farmacocinéticas e farmacodinâmicas decorrentes da ordem de infusão, as quais subsidiaram a construção de um quadro determinando o melhor sequenciamento para cada uma dessas combinações. Um fluxograma também foi produzido para, junto com o quadro, apoiar a SAE na perspectiva da prática de enfermagem oncológica baseada em evidência. Selecionar as sequências de infusão de antineoplásicos combinados representa nova estratégia conceitual para enfermeiros que administram protocolos de poliquimioterapia.

Descritores: Quimioterapia Combinada; Interações de Medicamentos; Neoplasias; Enfermagem Oncológica.

\footnotetext{
${ }^{1}$ Enfermeiro. Discente do Programa de Pós-Graduação Mestrado Profissional Enfermagem Assistencial da Universidade Federal Fluminense, nível Mestrado. Enfermeiro do Instituto Nacional de Câncer. Rio de Janeiro, RJ, Brasil. E-mail: angeloprimax@gmail.com.

${ }^{2}$ Enfermeira e Psicóloga, Doutora em Enfermagem. Professora Associada da Universidade Federal Fluminense. Rio de Janeiro, RJ, Brasil. E-mail: elianeramos.uff@gmail.com.

${ }^{3}$ Enfermeira, Doutora em Saúde Coletiva. Professor convidada da Universidade do Estado do Rio de Janeiro. Rio de Janeiro, RJ, Brasil. E-mail: carinne.mag@gmail.com.

${ }^{4}$ Enfermeira, Doutora em Ciências do Cuidado em Saúde. Professora convidada do curso de Residência em Farmácia Hospitalar da Universidade Federal Fluminense. Rio de Janeiro, RJ, Brasil. E-mail: bruna.barreto07@gmail.com.

${ }^{5}$ Enfermeira, Mestre em Enfermagem. Enfermeira do Instituto Nacional do Câncer. Rio de Janeiro, RJ, Brasil. E-mail: ticypinto@gmail.com.

${ }^{6}$ Enfermeira e Psicóloga, Doutora em Enfermagem e Doutora em Psicologia Social. Professora Associada da Universidade Federal Fluminense. Niterói, RJ, Brasil. E-mail: roserosauff@gmail.com.
}

Artigo recebido: 02/04/2018.

Artigo aprovado: 19/10/2018.

Artigo publicado: 31/12/2018.

\section{Como citar esse artigo:}

Mendonça AB, Pereira ER, Magnago C, Barreto BMF, Goes TRP, Silva RMCRA. Sequenciamento de infusão de antineoplásicos: contribuições para a prática de enfermagem oncológica baseada em evidência. Rev. Eletr. Enf. [Internet]. 2018 [acesso em: ];20:v20a51. Disponível em: https://doi.org/10.5216/ree.v20.52232. 


\section{INTRODUÇÃO}

Os quimioterápicos antineoplásicos constituem importante classe de fármacos utilizados no combate ao câncer. Por sua complexidade de administração, recomenda-se que sejam administrados exclusivamente por enfermeiros capacitados $^{(1)}$ que, ao instrumentalizar a sua prática clínica por meio de evidências científicas, pode manejar adequadamente os efeitos terapêuticos e tóxicos do tratamento através do adequado sequenciamento de infusão.

Estando sujeitos aos mesmos princípios de farmacocinética e farmacodinâmica que qualquer medicamento, os agentes antineoplásicos têm o mesmo potencial para interações medicamentosas. Considerando que os esquemas quimioterápicos empregam, em geral, duas ou mais drogas, a probabilidade de ocorrência de interações aumenta, agravando-se quanto maior for o número de medicamentos prescritos. Muitas destas interações têm uma importância clínica relativa, uma vez que poderão ser pouco lesivas ou até desejáveis. Outras, entretanto, poderão causar efeitos adversos graves, sendo a causa de morte de cerca de $4 \%$ dos doentes oncológicos ${ }^{(2-3)}$.

Por muito tempo combinou-se antineoplásicos com diferentes mecanismos de ação com a finalidade de superar a resistência a drogas e aumentar a dose-densidade de citostáticos. Entretanto, estudos sobre os mecanismos pelos quais a célula entra e prossegue no ciclo de divisão celular têm contribuído para a melhor associação de drogas nos protocolos atuais de quimioterapia. O conhecimento de vários pontos de verificação responsáveis por regular o ciclo celular permitiu melhorar a eficácia clínica de novos regimes terapêuticos e tornou evidente que a sequência de administração de drogas pode ser capaz de potencializar significativamente os efeitos terapêuticos sem aumentar a toxicidade clínica. Tais efeitos podem ser explicados por perturbações no ciclo celular induzidas pela quimioterapia ou por interações farmacodinâmicas entre os agentes combinados ${ }^{(4)}$.

Nesse sentido, e considerando que grande parte das interações medicamentosas desfavoráveis podem ser evitadas pelo correto planejamento da ordem de infusão, seu estudo, avaliação e conhecimento são imprescindíveis para a redução de erros, de morbimortalidade e de custos financeiros decorrentes de iatrogenias. Para aumentar a segurança de pacientes oncológicos em quimioterapia, um estudo recente demonstrou a eficácia de intervenções multiprofissionais que resguardam o cliente, como a implementação de código de barras antes da aplicação de citostáticos, prescrição eletrônica, conferência farmacêutica, uso de procedimentos padronizados, e um manual de interações medicamentosas que evite erros na sequência de administração(5).

Apesar da consistência dos resultados publicados para apoiar a ordem de infusão de determinados protocolos, faltam dados que forneçam bons níveis de evidência para outros, especialmente os que incluem medicamentos mais novos e menos comuns na prática. Na análise de sinergismo, antagonismo, efeitos terapêuticos e tóxicos, os resultados ainda são controversos para muitas das combinações de citostáticos, necessitando clarificação por meios de evidências científicas.

A Oncology Nurse Society (ONS), órgão que estabelece normas de segurança para administração de quimioterapia, ainda não dispõe de diretrizes específicas para o sequenciamento de antineoplásicos, mas ressalta a importância da criação de protocolos institucionais e padrões de assistência para o alcance de excelência nos cuidados prestados ${ }^{(6)}$. 
Pelo exposto e buscando subsidiar a tomada de decisão clínica, este artigo objetivou identificar as evidências cientificas disponíveis acerca das interações medicamentosas entre antineoplásicos decorrentes da ordem de infusão para, então, descrever a melhor sequência de aplicação, e discutir sua aplicabilidade para a Sistematização da Assistência de Enfermagem (SAE).

\section{MÉTODO}

Trata-se de revisão integrativa da literatura operacionalizada por meio de seis etapas: (1) formulação de questão de pesquisa; (2) estabelecimento dos procedimentos para a busca das evidências; (3) coleta dos dados; (4) avaliação dos dados; (5) análise crítica e interpretação; e (6) sumarização e apresentação dos resultados(7).

Esta revisão objetivou responder à seguinte questão: "Quais as interações medicamentosas entre antineoplásicos decorrentes da ordem de infusão?". Os critérios de inclusão delimitados para este estudo foram: estudos primários que tratassem de interações medicamentosas entre antineoplásicos, decorrentes da ordem de infusão, aprovados para uso humano no Brasil pela Agência Nacional de Vigilância Sanitária; publicados em português, inglês ou espanhol. Foram excluídas as publicações sem metodologia clara e reproduzível; as encontradas em mais de uma base; e as que abordavam o sequenciamento entre protocolos oncológicos e não entre drogas.

Entre os dias 18 de janeiro e 28 de fevereiro de 2018 foi realizada varredura nas bases de dados Medical Literature Analysis and Retrieval System Online (MEDLINE), via PUBMED; Biblioteca Virtual em Saúde (BVS); Cumulative Index of Nursing and Allied Health (CINAHL); e Literatura Latino-Americana e do Caribe em Ciências da Saúde (LILACS), sem recorte temporal definido. Também foram incluídos estudos por meio de referência cruzada.

Para definição do método de busca e identificação de descritores, adotou-se a estratégia PIO: (P) População/problema = neoplasias; (I) Intervenção = infusão de antineoplásicos; e (O) Outcomes (desfecho) = interações sinérgicas decorrentes da ordem de infusão. Os descritores e MeSH utilizados selecionados foram: Neoplasms; Drug Therapy; e Drug Interactions. Também foram adotadas as palavras-chave Chemotherapy e Sequence Administration. Os termos foram articulados por meio dos operadores booleanos OR e AND.

A seleção das publicações foi realizada por meio de três etapas. Na etapa de triagem foram aplicados filtros nas bases de dados para exclusão dos tipos de estudos não desejados e, na sequência, dos duplicados. Na etapa de elegibilidade aplicaram-se os critérios de inclusão e exclusão a partir da leitura dos títulos e resumos; e na etapa de inclusão foi realizada leitura do texto completo das publicações por dois revisores independentes que, juntos, acordaram a inclusão ou exclusão do material previamente selecionado na fase anterior, determinandose a amostra final. As publicações incluídas foram, então, analisadas, sumarizadas e discutidas na perspectiva dos objetivos propostos.

\section{RESULTADOS}

As buscas resultaram em 1.034 publicações; dessas, 57 (5,5\%) compuseram a amostra final (Figura 1).

Os 57 estudos analisados foram publicados na língua inglesa, em periódicos internacionais, entre os anos de 1973 e 2013, sendo que o maior número data do ano 2001 (n=6). A maioria foi divulgada em periódicos da área farmacêutica e oncológica, destacando-se o Journal Clinical Oncology (24,6\%) e Cancer Chemother Pharmacol 
(14\%). Não houve divulgação do tema em periódicos de enfermagem, sugerindo a necessidade de articular estudos de farmacocinética e farmacodinâmica à prática de administração de medicamentos, uma vez que evitar interações indesejáveis através do correto aprazamento é competência técnica e legal de enfermeiros.

Figura 1: Fluxograma do processo de seleção da literatura científica.

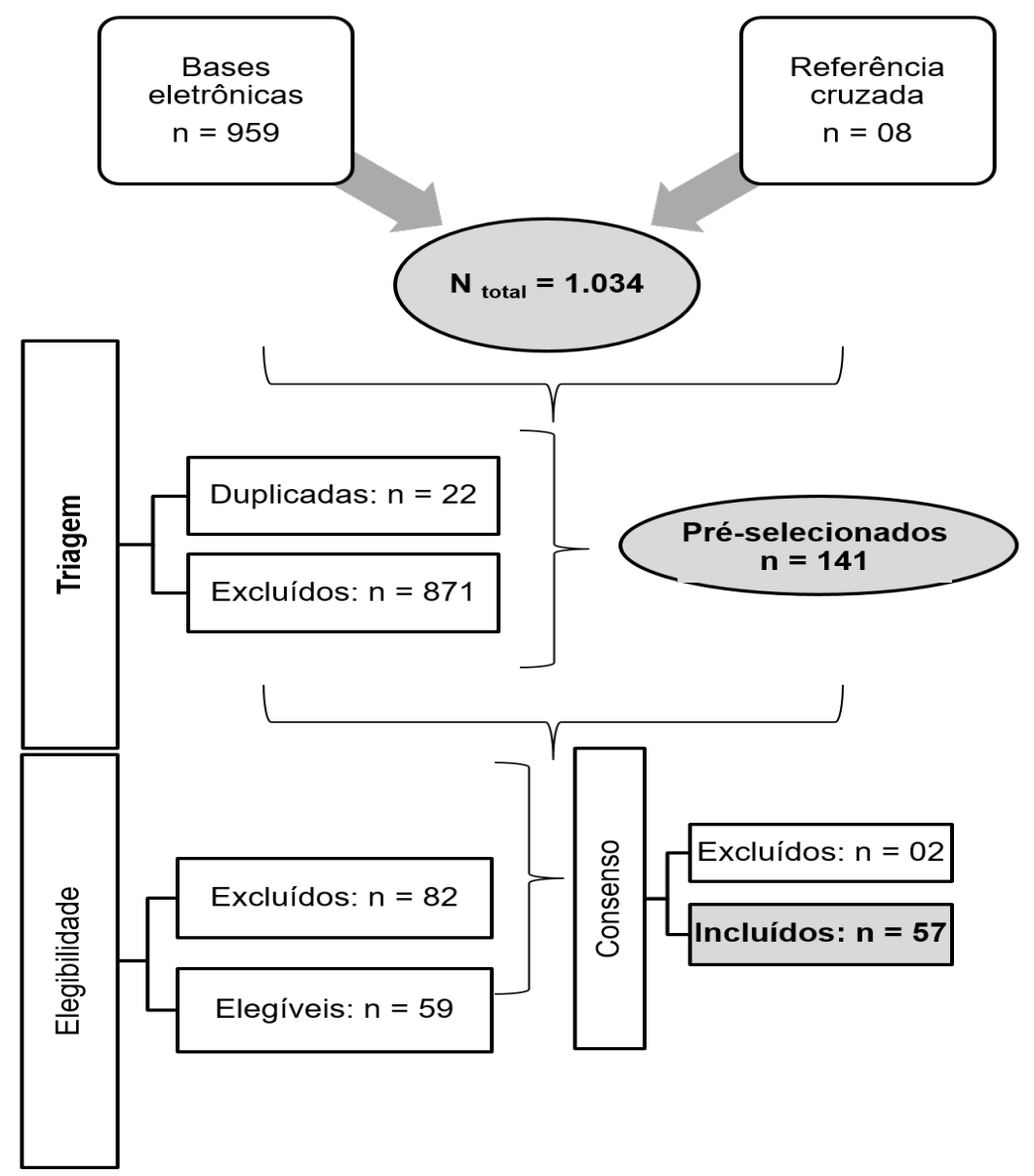

Quanto ao método aplicado para identificação de interações, 27 (47,4\%) estudos realizaram experimentos in vitro, classificado como evidência pouco robusta para sustentar recomendações específicas. O número considerável de interações documentadas através destes estudos, demonstrando sinergismo e antagonismo decorrentes da ordem de infusão, aponta a necessidade de investigação dos efeitos da sequência em ensaios clínicos bem delineados.

As razões farmacocinéticas e farmacodinâmicas para o sequenciamento de infusão de antineoplásicos e os impactos clínicos constatados estão apresentadas no Quadro 1, que pode ser disponibilizado para uso em centros de infusão como instrumento de apoio à tomada de decisão. 


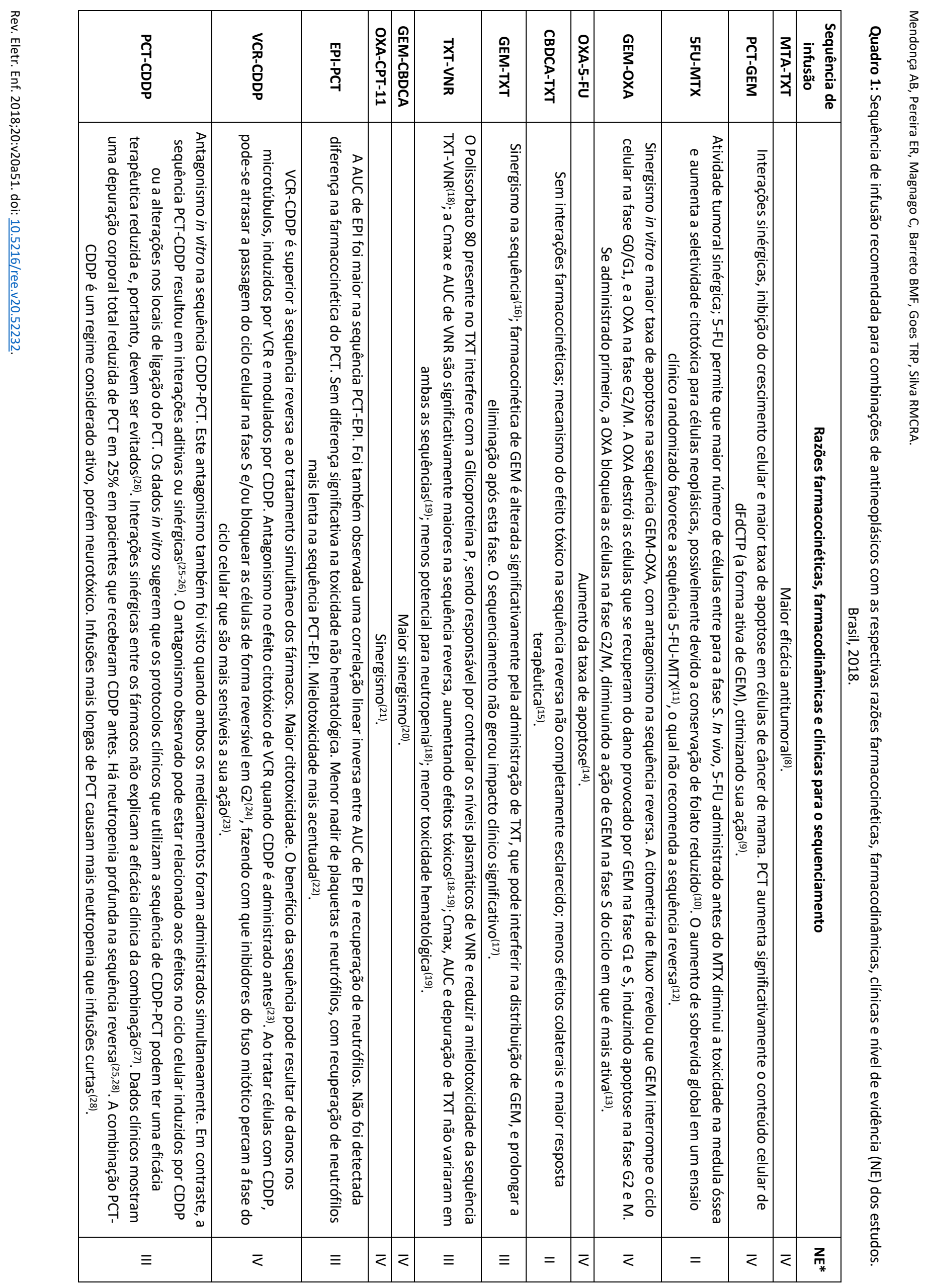




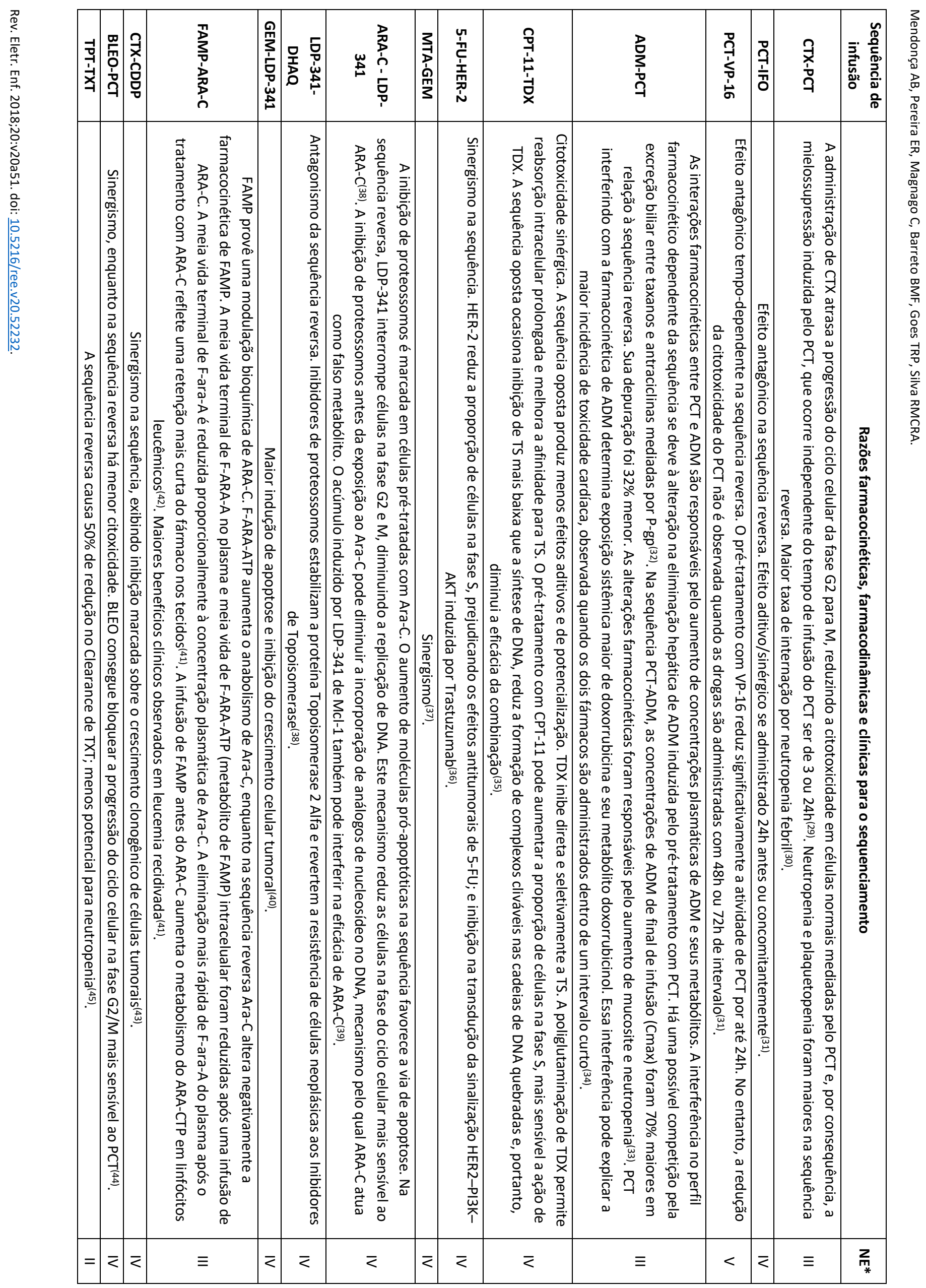




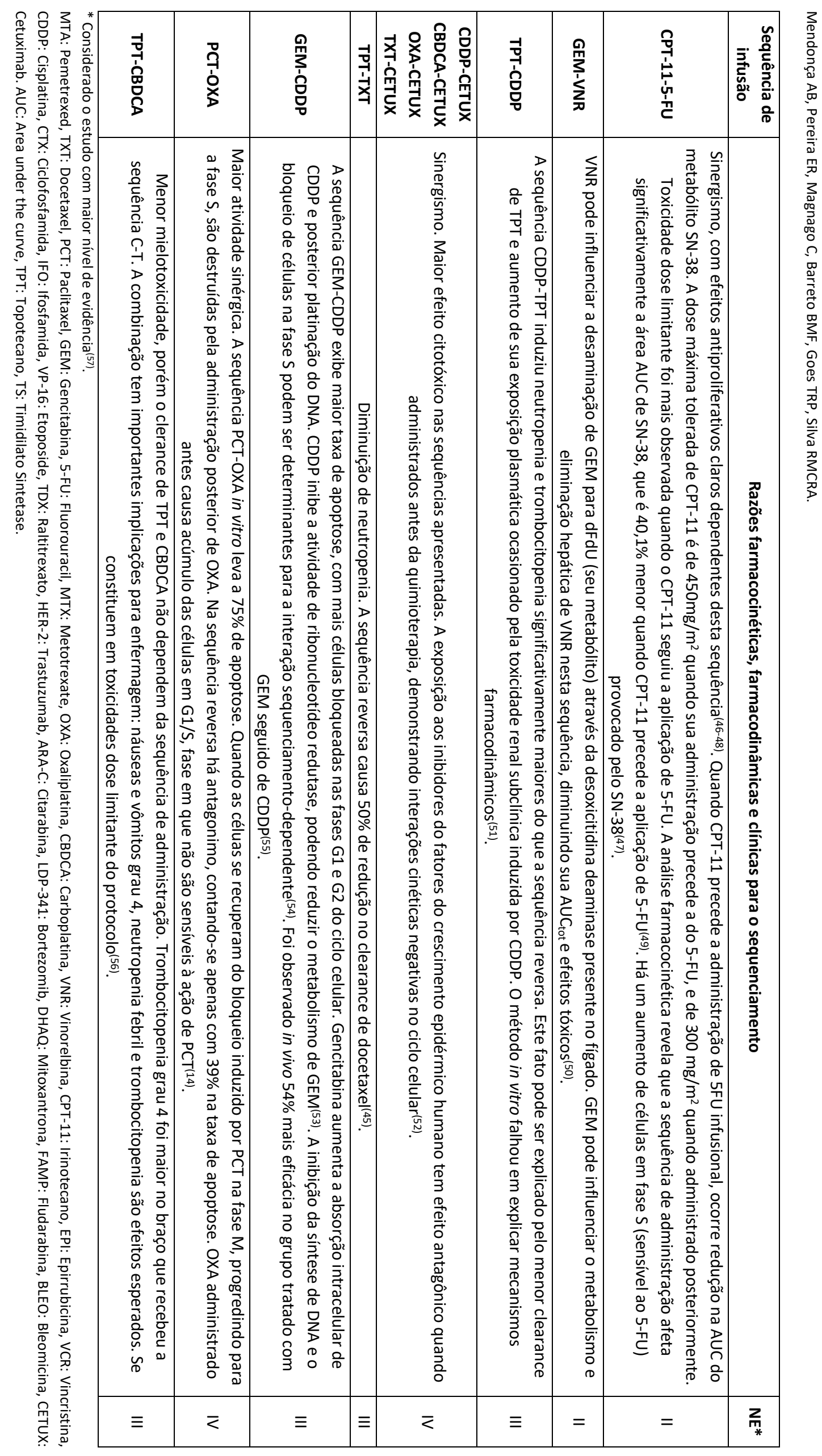


Para seis combinações de antineoplásicos, alguns estudos não encontraram diferenças nos perfis farmacocinéticos dependentes da ordem de infusão (Quadro 2).

Quadro 2: Combinações de antineoplásicos sem interações farmacocinéticas dependentes da ordem de infusão evidenciadas por alguns estudos. Brasil, 2018.

\begin{tabular}{|c|c|}
\hline Sequência & Resultados \\
\hline OXA-CPT11(58) $^{(58)}$ & $\begin{array}{r}\text { Não foram detectadas interaçães farmacocinéticas entre esses agentes. As principais toxicidades foram } \\
\text { neutropenia e diarreia tardia, independente da ordem de infusão. }\end{array}$ \\
\hline GEM-OXA(59) & $\begin{array}{r}\text { As sequências GEM-OXA e OXA-GEM mostraram um comportamento farmacocinético similar, sem } \\
\text { interação dependente de sequência. }\end{array}$ \\
\hline PCT-CBDCA & $\begin{array}{r}\text { (60-62) } \\
\text { A farmacocinética da carboplatina não é alterada pelo pré-tratamento com PCT na dose padrão. Interação } \\
\text { farmacocinética não é responsável pela menor toxicidade da combinação. Neutropenia é o principal efeito, } \\
\text { anemia é frequente, e trombocitopenia tem menor incidência. }\end{array}$ \\
\hline BEVA-CPT-11(63) & $\begin{array}{r}\text { O BEVA não afeta a farmacocinética de CPT-11. Uma variedade de relações farmacogenéticas podem } \\
\text { influenciar a farmacocinética do CPT-11 e sua toxicidade. }\end{array}$ \\
\hline CDDP-CPT-11(64) & $\begin{array}{r}\text { Nenhuma alteração na farmacocinética resulta da ordem de administração. A combinação fornece um } \\
\text { regime prático e bem tolerado, com potencial de maximizar sinergia entre os agentes. }\end{array}$ \\
\hline ADM-PCT(65) & $\begin{array}{c}\text { A ordem de infusão não afeta a farmacocinética e toxicidade. Altas taxas de resposta completa e } \\
\text { insuficiência cardíaca congestiva são uma expressão de efeitos terapêuticos e tóxicos desta combinação. }\end{array}$ \\
\hline
\end{tabular}

Legenda: OXA: Oxaliplatina, CPT11: Irinotecano, GEM: Gencitabina, PCT: Paclitaxel, CBDCA: Carboplatina, BEVA: Bevacizumab, CDDP: Cisplatina, ADM: Doxorrubicina.

Para as sequências Bevacizumab-Irinotecano (BEVA-CPT-11)(63), Cisplatina-Irinotecano (CDDP-CPT-11) ${ }^{(64) \text {, }}$ Oxaliplatina-Irinotecano (OXA-CPT-11)(58) e Paclitaxel-Carboplatina (PCT-CBDCA) ${ }^{(60-62)}$ não houve repercussões clínicas em termos de toxicidade ou benefício terapêutico. No que diz respeito a sequência PCT-CBDCA, apesar deste estudo não ter encontrado evidências científicas para sustentá-la, há, na literatura, ampla recomendação para esta ordem de infusão. As justificativas se apoiam no risco de neutropenia que análogos de platina provocam quando antecedem taxanos na sequência, e pelos riscos de lesões secundárias ao extravasamento de vesicantes, como o PCT ${ }^{(66)}$.

Os resultados controversos ${ }^{(32-34,65)}$ para a sequência Doxorrubicina-Paclitaxel (ADM-PCT) (Quadro 1 e 2) se devem, em parte, à pequena amostra estudada e à variabilidade genética observada entre indivíduos. Polimorfismos genéticos são responsáveis pela diversidade na carga de enzimas indutoras, metabolizadoras e transportadores de influxo, ocasionando diferentes respostas e graus de interação entre fármacos. É sugerido que fatores genéticos podem contribuir com 20 a 95\% na variabilidade da eficácia terapêutica e tóxica de ADM. Destarte, a compreensão de vias metabólicas de ADM tem possibilitado correlações farmacogenéticas, farmacocinéticas e farmacodinâmicas, proporcionando individualização terapêutica ${ }^{(67-68)}$. Outrossim, estudos têm demonstrado que tempos de infusão mais prolongados de ADM podem não só se refletir em alterações de parâmetros farmacocinéticos, mas também em cardiotoxicidade, interferindo nos resultados dos estudos analisados. Neste contexto, interações farmacocinéticas com a combinação foram melhor caracterizadas em estudos com PCT em infusão de $24 \mathrm{~h}$, em detrimento de infusão de $3 \mathrm{~h}^{(69)}$. As publicações revisadas advogando a sequência ADM-PCT foram claramente superiores às que demonstram ausência de interações para a sequência. Dada a magnitude e consequências da elevação nas concentrações plasmáticas de doxorrubicina ${ }^{(70-72)}$, este artigo recomenda ADM-PCT. 
Para a sequência Gencitabina-Oxaliplatina (GEM-OXA), os resultados favoráveis in vitro ${ }^{(13)}$ (Quadro 1) não se traduziram em maior benefício clínico in vivo(59) (Quadro 2), reforçando cautela na sua interpretação e uso prático.

\section{DISCUSSÃO}

Administrar quimioterapia na ordem errada é considerado um erro de medicação, e se constitui na causa mais comum de erro $(50,5 \%)$ entre enfermeiros que atuam em centros de quimioterapia ambulatorial(73). Essa constatação ratifica a relevância do planejamento da ordem de infusão apoiando-se nos melhores níveis de evidência para a tomada de decisão, tal como apregoa a prática baseada em evidências (PBE).

Com o intuito de nortear a prática de sequenciamento de infusão realizada por enfermeiros, segundo a PBE, foi elaborado o fluxograma apresentado na Figura 2.

Figura 2: Fluxograma de apoio à prática de sequenciamento de infusão de antineoplásicos baseada em evidências. Brasil, 2018.

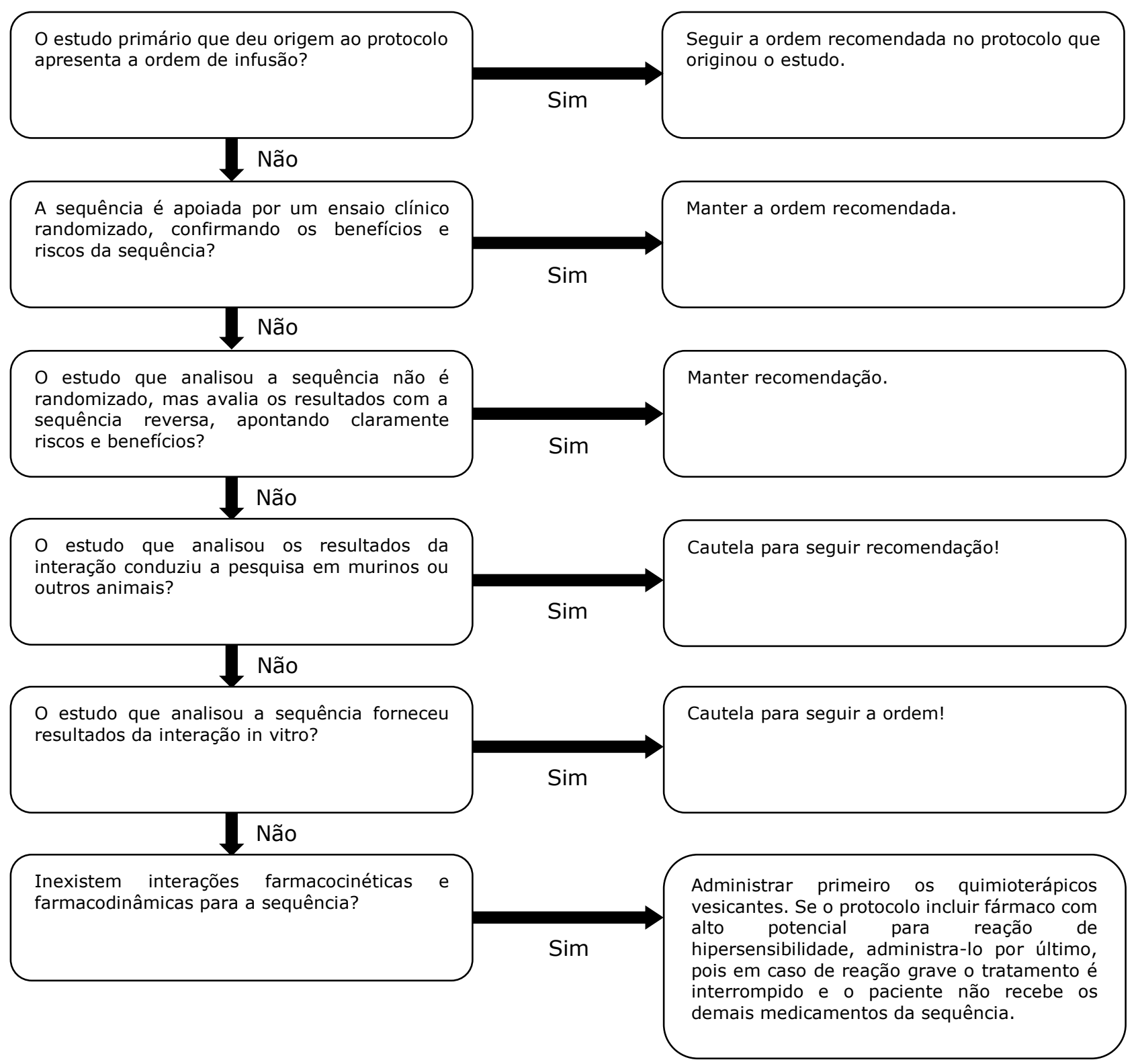


O quadro de sequenciamento de antineoplásicos deve ser avaliado junto ao fluxograma (Figura 2), pois determinados protocolos de quimioterapia já estabelecem a sequência e o tempo de infusão dos fármacos. São consagrados por derivar de estudos multicêntricos fase III ou IV, acompanhando grandes populações de pacientes por um longo período. Alterações em seu fluxo podem comprometer a eficácia do tratamento em termos de benefício clínico, sobrevida global e segurança.

Para reduzir os riscos de alteração da sequência prevista nos protocolos de quimioterapia, publicações contendo uma descrição precisa do regime devem ser consultadas antes da administração das drogas, como manuais de oncologia e handbooks. Se a ordem de aplicação não se encontra explícita para o regime em questão, o enfermeiro torna-se responsável por clarificar a sequência como parte do processo de enfermagem. Para isso recomenda-se o processo de verificação de enfermagem, por meio do qual enfermeiros peritos fazem dupla conferência das prescrições de antineoplásicos, checando a combinação correta dos fármacos, o cálculo de superfície corporal, o intervalo entre ciclos, a adequação da dose, o padrão de diluição e a ordem e o tempo de infusão $0^{(74)}$.

Na ausência de interações farmacocinéticas e farmacodinâmicas, a literatura recomenda a administração de quimioterápicos vesicantes antes dos não vesicantes em veias periféricas, como apontado no fluxograma (Figura 2). Esta estratégia diminui o risco de extravasamento, pois no início do tratamento o vaso encontra-se íntegro, isto é, menos afetado por reações locais induzidas por outros agentes, como eritema, dor e prurido(75). Em se tratando de combinações com mais de um agente vesicante, os riscos e gravidade de lesões secundárias ao extravasamento ${ }^{(76-77)}$ justificam a administração de vesicantes que se ligam ao DNA primeiro, seguido pelos que não se ligam e, por fim, pelos quimioterápicos neutros.

Embora tenham sido analisadas as melhores evidências atualmente disponíveis para a construção do Quadro 1, cabe ressaltar que, até o momento, não foram encontradas revisões sistemáticas e metanálises sobre a ordem de infusão de antineoplásicos, estudos esses considerados como de maior relevância para a tomada de decisão clínica, segundo a PBE.

No que se refere à força de evidência de estudos in vitro, muitos analisaram o efeito da sequência entre antineoplásicos através da incubação de linhagens celulares em fármacos por períodos de $24 \mathrm{~h}$ ou mais. Estes resultados podem não ser consistentes para apoiar a prática de sequenciamento de medicamentos administrados no mesmo dia em seres humanos. Apesar dos fortes indícios de efeitos no ciclo celular induzidos pela ordem de infusão demonstrados nestes estudos, o perfil de ação farmacológica da droga pode variar consideravelmente dadas as alterações de parâmetros farmacocinéticos observados in vivo. Dentre estes parâmetros citam-se a ligação do fármaco a proteínas plasmáticas, distribuição, metabolismo, eliminação, meia vida e tempo de ação no organismo.

Estudos com interações medicamentosas in vivo, demonstrando alterações em parâmetros farmacocinéticos, apresentam relevância clínica superior, pois determinam com maior precisão a concentração da droga nos vários compartimentos do organismo, seu tempo de chegada até o sítio de ação, duração e magnitude do efeito terapêutico/tóxico, e tempo para eliminação.

Embora estudos in vitro possam ter limitações para apoiar determinada sequência na prática clínica de enfermeiros, na ausência de interações documentadas in vivo e em se tratando de agentes neutros (sem caráter 
vesicante ou irritante), estes podem fornecer uma justificativa razoável para estabelecer a ordem de infusão. As descobertas desse tipo de estudo têm importantes implicações para o desenho de protocolos atuais de quimioterapia, fornecendo informações moleculares importantes sobre o efeito da interação de drogas no nível celular. Sua revisão cuidadosa demonstra um largo número de substratos in vivo para fármacos antineoplásicos, inibidores e indutores de enzimáticos, responsáveis pelo metabolismo e interações medicamentosas ${ }^{(78)}$.

Métodos in vitro têm avançado cada vez mais com o uso de cultura de células em 3D. Além do estudo de toxicidade relacionada aos fármacos, adquirem significado especial na avaliação de estratégias de administração de drogas, oferecendo um vasto potencial em termos de especificidade na terapia alvo-dirigida, interações medicamentosas no nível celular e o papel de excipientes nas interações. Esses novos modelos oferecem a possibilidade de investigar terapias avançadas, incluindo medicamentos genéticos e formulações contendo nanopartículas; e prometem resultados consistentes em termos de sensibilidade às drogas e efeitos tóxicos. Entre os desafios futuros estão as correlações mais robustas de métodos in vitro-in vivo para melhorar a confiança e garantir um uso seguro(79).

Estudos in vitro apresentam, portanto, o seu valor, e não devem ser descartados para a tomada de decisão. A falta de evidências de alta qualidade para algumas sequências de antineoplásicos não impossibilita a tomada de decisões segundo a PBE. Nessa situação, o que é requerido é a melhor evidência disponível e não a melhor evidência possível(80).

A grande variabilidade e complexidade de combinações entre drogas e de regimes clínicos em curso, além da rápida incorporação de protocolos de pesquisa aos tratamentos clínicos convencionais, têm exigido atualização frequente de médicos, enfermeiros e farmacêuticos na busca por melhores evidências.

\section{Implicações para a Sistematização da Assistência de Enfermagem}

Os eventos adversos mais encontrados nos estudos in vivo dependentes do sequenciamento de infusão nesta revisão foram a neutropenia ${ }^{(18,25,28,30,33,45,51,56)}$ e a trombocitopenia ${ }^{(51,56)}$, os quais estão intimamente relacionados aos diagnósticos de enfermagem 'risco para infecção' e 'risco para sangramento'. Outros efeitos tóxicos implicados na ordem de administração foram a diarreia ${ }^{(49)}$, a hepatotoxicidade ${ }^{(50)}$, náuseas ${ }^{(49)}$, $\operatorname{vômitos}^{(56)}$, mucosite $^{(33)}$ e cardiotoxicidade ${ }^{(34)}$, os quais também estão associados a diagnósticos de enfermagem previstos no sistema de classificação da NANDA Internacional| ${ }^{(81)}$.

Em serviços ambulatoriais de oncologia, os cuidados prestados devem ter como foco as necessidades do indivíduo, utilizando-se os diagnósticos de enfermagem como taxonomia padronizada. Esta ferramenta assistencial fornece apoio para a tomada de decisão e direciona a seleção de intervenções mais eficazes em melhorar os padrões de resposta dos pacientes em tratamento antineoplásico ${ }^{(82)}$.

Um estudo que analisou a natureza e a classificação das intervenções de enfermagem em um ambulatório de quimioterapia adulto constatou um predomínio de ações direcionadas ao aconselhamento nutricional, não sendo reportado o diagnóstico de enfermagem 'risco para infecção', tampouco intervenções para a sua prevenção/controle e programação da ordem de infusão dos medicamentos ${ }^{(83)}$. Estes achados chamam atenção, pois esta lacuna na assistência aumenta a vulnerabilidade de pacientes às complicações infecciosas, além de outros efeitos derivados de erros na ordem de administração dos fármacos. Por meio do planejamento efetivo da 
ordem de infusão, o enfermeiro pode, em sua prática, reduzir não somente a incidência e severidade destas complicações, mas também os ajustes de dose e os atrasos no tratamento provocados por mielodepressão profunda e prolongada.

Destarte, o enfermeiro que administra quimioterapia antineoplásica tem a responsabilidade de supervisionar e orientar os cuidados destinados à prevenção e ocorrência de infecção. Tais intervenções devem ser planejadas na terceira etapa do processo de enfermagem ${ }^{(84)}$, tendo em conta o potencial mielotóxico individual das drogas, período de NADIR e de recuperação medular prevista para o protocolo em questão(85). Na quarta etapa do processo de enfermagem, designada como fase de implementação da assistência (Prescrição de Enfermagem) ${ }^{(84)}$, estudos têm considerado como intervenções prioritárias a pacientes idosos, portadores de câncer de mama e neoplasias hematológicas de alto grau, o controle da contagem de neutrófilos, administração profilática de fatores de crescimento hematopoético ${ }^{(85)}$ e infusão dos quimioterápicos, seguindo uma ordem que reduza o risco de neutropenia febril. A última intervenção é capaz de proporcionar melhor resposta terapêutica, que advém do sinergismo bioquímico e farmacodinâmico entre os agentes envolvidos na sequência.

Pelo exposto, o planejamento da ordem de infusão deve ser realizado com a mesma seriedade com que se escolhe o dispositivo para o acesso vascular e se efetuam cuidados na inserção do cateter. Tais ações visam a prevenção de lesões de pele secundária a extravasamentos e redução dos efeitos tóxicos provenientes de um sequenciamento inadequado.

Recomendações para melhorar a segurança e diminuir erros na administração de quimioterapia incluem a criação de processos padronizados e a adesão rigorosa a políticas e procedimentos rotineiramente implantados, com o objetivo de garantir qualidade em todas as fases do processo ${ }^{(6,74)}$.

Nesse contexto, o quadro de sequenciamento de antineoplásicos (Quadro 1) pode subsidiar o estabelecimento de padrões de ordem de infusão e, por consequência, melhorar a eficiência do trabalho de farmacêuticos e enfermeiros, reduzindo tempo de espera em ambulatórios de quimioterapia com alto fluxo de pacientes; evitar o preparo antecipado de drogas que não serão administradas primeiramente; e diminuir os riscos de erros por sequenciamento incorreto, omissão de medicamentos, desvios de protocolo e atrasos no tratamento.

Para melhorar a segurança do paciente, a equipe deve partilhar das mesmas informações e condutas sobre as implicações clínicas envolvidas na ordem de infusão. A consciência dos riscos envolvidos nesta ação de enfermagem alerta para a necessidade de gestores realçarem as boas práticas ao elaborarem protocolos institucionais de administração de citostáticos, parte da SAE. Diante desta nova realidade, reitera-se que o sequenciamento incorreto de antineoplásicos se constitui risco para a integridade física e sobrevida dos pacientes sob regime de quimioterapia, requerendo a presença de profissionais de enfermagem altamente qualificados e detentores do conhecimento baseado em evidências para conduzir o processo de enfermagem ${ }^{(85)}$.

\section{CONCLUSÃO}

O tratamento quimioterápico e seu aprazamento requerem do enfermeiro um profundo conhecimento dos mecanismos moleculares, farmacodinâmicos e farmacocinéticos subjacentes a esta ação. Selecionar as sequências de infusão de drogas combinadas, baseando-se no entendimento destes mecanismos, representa uma nova estratégia conceitual para enfermeiros que administram protocolos de poliquimioterapia. 
O quadro elaborado neste estudo, que indica o melhor sequenciamento, é um instrumento de fácil consulta para enfermeiros e farmacêuticos, podendo ser disponibilizado nos serviços de infusão, contribuindo para a prevenção e redução de erros derivados do sequenciamento de infusão inadequado. Visa-se assegurar aos pacientes menor toxicidade e maior benefício clínico em função de uma melhor interação sinérgica entre os fármacos. Nesse sentido, é um importante instrumento para a SAE, que subsidia a gerência de risco para um cuidado mais seguro.

Ressalta-se que uma limitação deste estudo se refere à ausência de estudos com altos níveis de evidência, capazes de reunir as sequências de administração para os principais protocolos utilizados na prática clínica. De igual maneira, são poucos os estudos que abordem a SAE no contexto da poliquimioterapia. Pelo exposto, novos estudos que busquem preencher essas lacunas do conhecimento são requeridos para apoiar a PBE e, assim, assegurar uma assistência mais eficaz e segura.

\section{Financiamento:}

"O presente trabalho foi realizado com apoio da Coordenação de Aperfeiçoamento de Pessoal de Nível Superior Brasil (CAPES) - Código de Financiamento 001.

\section{REFERÊNCIAS}

1. Resolução do Conselho Federal de Enfermagem no 0569/2018 (BR) [Internet]. Aprova o Regulamento Técnico da Atuação dos Profissionais de Enfermagem em Quimioterapia Antineoplásica. Brasília, 19 fev. 2018 [acesso em: 01 abr 2018]. Disponível em: http://www.cofen.gov.br/resolucao-cofen-no-0569-2018 60766.html.

2. Lima TAM, Godoy MF. Interações medicamentosas em prescrições para idosos hospitalizados com Síndrome Coronariana Aguda. Rev Eletr Enf [Internet]. 2017 [cited 2018 Mar 01]; 19:a24. Available from: http://dx.doi.org/10.5216/ree.v19.42764.

3. van Leeuwen RWF, Brundel DHS, Neef C, van Gelder T, Mathijssen RHJ, Burger DM et al. Prevalence of potential drug-drug interactions in cancer patients treated with oral anticancer drugs. Br J Cancer [Internet]. 2013 [cited 2018 Mar 01];108(5):1071-8. Available from: http://www.nature.com/doifinder/10.1038/bjc.2013.48.

4. Shah MA, Schwartz GK. The relevance of drug sequence in combination chemotherapy. Drug Resist Updat [Internet]. 2000 [cited 2018 Mar 01];3(6):335-56. Available from: http://dx.doi.org/10.1054/drup.2000.0165.

5. Huertas-Fernández MJ, Martínez-Bautista MJ, Rodríguez-Mateos ME, Zarzuela-Ramírez T, Baena-Cañada JM. Implementation of safeguards to improve patient safety in chemotherapy. Clin Transl Oncol. [Internet]. 2017 [cited 2018 Sept 01];19(9):1009-106. Available from: http://dx.doi.org/10.1007/s12094-017-1645-y.

6. Neuss MN, Gilmore TR, Belderson KM, Billett AL, Conti-Kalchik T, Harvey BE et al. 2016 Updated American Society of Clinical Oncology/Oncology Nursing Society Chemotherapy Administration Safety Standards, Including Standards for Pediatric Oncology. J Oncol Pract [Internet]. 2016[cited 2018 Mar 28];12(12):1262-71. Available from:

http://ascopubs.org/doi/10.1200/JOP.2016.017905.

7. Cooper HM. The integrative research review: a systematic approach. Beverly Hills (CA): Sage Pulications; 1984.

8. Kano Y, Tanaka M, Akutsu M, Mori K, Yazawa Y, Mano H et al. Schedule-dependent synergism and antagonism between pemetrexed and docetaxel in human lung cancer cell lines in vitro. Cancer Chemother Pharmacol [Internet]. 2009[cited 2018 Mar 28];64(6):1129-37. Available from: http://dx.doi.org/10.1007/s00280-009-0974-0.

9. Oliveras-Ferraros C, Vazquez-Martin A, Colomer R, De Llorens R, Brunet J, Menendez JA. Sequence-dependent synergism and antagonism between paclitaxel and gemcitabine in breast cancer cells: The importance of scheduling. Int J Oncol [Internet]. 2008[cited 2018 Mar 28];32(1):113-20. Available from: https://doi.org/10.3892/ijo.32.1.113.

10. Das JR, Fryar-Tita EB, Zhou Y, Green S, Southerland WM, Bowen D. Sequence-dependent administration of 5-fluorouracil maintains methotrexate antineoplastic activity in human estrogen-negative breast cancer and protects against methotrexate cytotoxicity in human bone marrow. Anticancer Res [Internet]. 2007[cited 2018 Mar 28];27(6 B):3791-9. Available from:

http://ar.iiarjournals.org/content/27/6B/3791.long.

11. Mackintosh JF, Coates AS, Tattershall MH, Swanson C. Chemotherapy of advanced head and neck cancer: updated results of a randomized trial of the order of administration of sequential methotrexate and 5-fluorouracil. Med Pediatr Oncol. 1998;16:304-7. 
12. Coates AS, Tattersall MHN, Swanson C. Combination therapy with methotrexate and 5-fluorouracil: A prospective randomized clinical trial of order of administration. J Clin Oncol. 1984[cited 2018 Mar 28];2(7):756-61. Available from:

https://doi.org/10.1200/JCO.1984.2.7.756.

13. Makiyama A, Qin B, Uchino K, Shibata Y, Arita S, Isobe T et al. Schedule-dependent synergistic interaction between gemcitabine and oxaliplatin in human gallbladder adenocarcinoma cell lines. Anticancer Drugs [Internet]. 2009 [cited 2018 Mar 28];20(2):12330. Available from: https://doi.org/10.1097/CAD.0b013e3283218080.

14. Tanaka R, Ariyama H, Qin B, Takii Y, Baba E, Mitsugi K et al. In vitro schedule-dependent interaction between paclitaxel and oxaliplatin in human cancer cell lines. Cancer Chemother Pharmacol [Internet]. 2005 [cited 2018 Mar 26];55(6):595-601. Available from: https://doi.org/10.1007/s00280-004-0966-z.

15. Ando M, Saka H, Ando Y, Minami H, Kuzuya T, Yamamoto M et al. Sequence effect of docetaxel and carboplatin on toxicity, tumor response and pharmacokinetics in non-small-cell lung cancer patients: a phase I study of two sequences. Cancer Chemother Pharmacol [Internet]. 2005 [cited 2018 Mar 27];55(6):552-8. Available from: https://doi.org/10.1007/s00280-004-0921-z. 16. Leu KM, Ostruszka L, Shewach D, Zalupski M, Sondak V, Biermann JS et al. Laboratory and clinical evidence of synergistic cytotoxicity of sequential treatment with gemcitabine followed by docetaxel in the treatment of sarcoma. J Clin Oncol [Internet]. 2004 [cited 2018 Mar 22];22(9):1706-12. Available from: https://doi.org/10.1200/JCO.2004.08.043.

17. Dumez H, Louwerens M, Pawinsky A, Planting ASTh, Jonge MJA, Van Oosterom AT et al. The impact of drug administration sequence and pharmacokinetic interaction in a phase I study of the combination of docetaxel and gemcitabine in patients with advanced solid tumors. Anticancer Drugs [Internet]. 2002 [cited 2018 Mar 22];13(6):583-93. Available from:

https://insights.ovid.com/pubmed?pmid=12172503.

18. Airoldi M, Cattel L, Pedani F, Marchionatti S, Tagini V, Bumma C et al. Clinical data and pharmacokinetics of a docetaxelvinorelbine combination in anthracycline resistant/relapsed metastatic breast cancer. Acta Oncol. 2003;42(3):186-94.

19. Cattel L, Recalenda V, Airoldi M, Tagini V, Arpicco S, Brusa P et al. A sequence-dependent combination of docetaxel and vinorelbine: pharmacokinetic interactions. Farmaco [Internet]. 2001 [cited 2018 Mar 22];56(10):779-84. Available from:

https://doi.org/10.1016/S0014-827X(01)01138-7.

20. Edeiman MJ, Quam H, Mullins B. Interactions of gemcitabine, carboplatin and paclitaxel in molecularly defined non-small-cell lung cancer cell lines. Cancer Chemother Pharmacol. 2001;48(2):141-4.

21. Qin B, Tanaka R, Shibata Y, Arita S, Ariyama H, Kusaba H, Baba E, Harada MNS. In-vitro schedule-dependent interaction between oxaliplatin and 5-fluorouracil in human gastric cancer cell lines. Anticancer Drugs. 2006;17(4):445-43.

22. Venturini M, Lunardi G, Del Mastro L, Vannozzi MO, Tolino G, Numico G et al. Sequence effect of epirubicin and paclitaxel treatment on pharmacokinetics and toxicity. J Clin Oncol. 2000;18(10):2116-25.

23. Rowinsky EK, Citardi MJ, Noe DA, Donehower RC. Sequence-dependent cytotoxic effects due to combinations of cisplatin and the antimicrotubule agents taxol and vincristine. J Cancer Res Clin Oncol. 1993;119(12):727-33.

24. Fraval HNA, Roberts JJ. G1 phase chinese hamster V79-379A cells are inherently more sensitive to platinum bound to their dna than mid S phase or asynchronously treated cells. Biochem Pharmacol. 1979;28(10):1575-80.

25. Rowinsky EK, Gilbert MR, McGuire WP, Noe DA, Grochow LB, Forastiere AA, et al. Sequences of taxol and cisplatin: a phase I and pharmacologic study. J Clin Oncol. 1991;9(9):1692-703.

26. Vanhoefer U, Harstrick A, Wilke H, Schleucher N, Walles H, Schroder J et al. Schedule-dependent antagonism of paclitaxel and cisplatin in human gastric and ovarian carcinoma cell lines in vitro. Eur J Cancer [Internet]. 1995 [cited 2018 Mar 16];31A(1):92-7. Available from: https://doi.org/10.1016/0959-8049(94)00440-G.

27. Levasseur LM, Greco WR, Rustum YM, Slocum HK. Combined action of paclitaxel and cisplatin against wildtype and resistant human ovarian carcinoma cells. Cancer Chemother Pharmacol [Internet]. 1997 [cited 2018 Mar 16];40(6):495-505. Available from: https://doi.org/10.1007/s002800050693.

28. Wasserheit C, Frazein A, Oratz R, Sorich J, Downey A, Hochster $\mathrm{H}$ et al. Phase II trial of paclitaxel and cisplatin in women with advanced breast cancer: an active regimen with limiting neurotoxicity. J Clin Oncol. 1996;13(7):1993-9.

29. Kennedy MJ, Zahurak ML, Donehower RC, Noe D, Grochow LB, Sartorius S et al. Sequence-dependent Hematological Toxicity Doublet' Associated with the Paclitaxel/Cyclophosphamide. Clin Cancer Res [Internet]. 1998 [cited 2018 Mar 14];4(2):349-56.

Available from: http://clincancerres.aacrjournals.org/content/4/2/349.long.

30. Kennedy MJ, Zahurak ML, Donehower RC, Sartorius S, Chen TL, Bowling K et al. Phase I and pharmacologic study of sequences of paclitaxel and cyclophosphamide supported by granulocyte colony-stimulating factor in women with previously treated metastatic breast cancer. J Clin Oncol [Internet]. 1996 [cited 2018 Mar 14];14(3):783-91. Available from:

https://doi.org/10.1200/JCO.1996.14.3.783.

31. Klaassen U, Harstrick A, Schleucher N, Vanhoefer U, Schroder J, Wilke H et al. Activity- and schedule-dependent interactions of pacditaxel, etoposide and hydroperoxy-ifosfamide in cisplatin-sensitive and -refractory human ovarian carcinoma cell lines. Br J Cancer. 1996;74:224-8.

32. Gianni L, Viganò L, Locatelli A, Capri G, Giani A, Tarenzi EBG. Human pharmacokinetic characterization and in vitro study of the interaction between doxorubicin and paclitaxel in patients with breast cancer. J Clin Oncol. 1997;15(5):1906-15. 
33. Holmes FA, Madden T, Newman RA, Valero V, Theriault RL, Fraschini G et al. Sequence-dependent alteration of doxorubicin pharmacokinetics by paclitaxel in a phase I study of paclitaxel and doxorubicin in patients with metastatic breast cancer. J Clin Oncol. 1996;14(10):2713-21.

34. Moreira A, Lobato R, Morais J, Silva S, Ribeiro J, Figueira A et al. Influence of the interval between the administration of doxorubicin and paclitaxel on the pharmacokinetics of these drugs in patients with locally advanced breast cancer. Cancer Chemother Pharmacol. 2001;48(4):333-7.

35. Aschele C, Baldo C, Sobrero AF, Debernardis D, Bornmann WG, Bertino JR. Schedule-dependent synergism between raltitrexed and irinotecan in human colon cancer cells in vitro. Clin Cancer Res. 1998;4(5):1323-30.

36. Li XL, Yi SQ, Xu JM, Zhang Y, Yingying-Feng, Chen W et al. The sequence-dependent cytotoxic effect of trastuzumab in combination with 5-fluorouracil or cisplatin on gastric cancer cell lines. Cancer Invest [Internet]. 2010 [cited 2018 Mar 20];28(10):1038-47. Available from: https://doi.org/10.3109/07357907.2010.483512.

37. Nagai S, Takenaka K, Sonobe M, Wada H, Tanaka F. Schedule-dependent synergistic effect of pemetrexed combined with gemcitabine against malignant pleural mesothelioma and non-small cell lung cancer cell lines. Chemotherapy [Internet]. 2008 [cited 2018 Mar 20];54(3):166-75. Available from: https://doi.org/10.1159/000140360.

38. Hutter $\mathrm{G}$, Rieken $\mathrm{M}$, Pastore A, Weigert $\mathrm{O}$, Zimmermann $\mathrm{Y}$, Weinkauf $\mathrm{M}$ et al. The proteasome inhibitor bortezomib targets cell cycle and apoptosis and acts synergistically in a sequence-dependent way with chemotherapeutic agents in mantle cell lymphoma. Ann Hematol [Internet]. 2012 [cited 2018 Mar 25];91(6):847-56. Available from: https://doi.org/10.1007/s00277-011-1377-y. 39. Weigert O, Pastore A, Rieken M, Lang N, Hiddemann W, Dreyling M. Sequence-dependent synergy of the proteasome inhibitor bortezomib and cytarabine in mantle cell lymphoma. Leukemia. 2007;21(3):524-8.

40. Fahy BN, Schlieman MG, Virudachalam S, Bold RJ. Schedule-dependent molecular effects of the proteasome inhibitor bortezomib and gemcitabine in pancreatic cancer. J Surg Res. 2003;113(1):88-95.

41. Kemenal A, Gandhi V, Shewach DS, Keatingt M, Plunkettl W. Inhibition of fludarabine metabolism by arabinosyleytosine during therapy. Cancer Chemother Pharmacol. 1992;31:193-9.

42. Gandhi V, Kemena A, Keating MJ, Plunkett W. Fludarabine Infusion Potentiates Arabinosylcytosine Metabolism in Lymphocytes of Patients with Chronic Lymphocytic Leukemia Fludarabine Infusion Potentiates Arabinosylcytosine Metabolism in Lymphocytes of Patients with Chronic Lymphocytic Leukemia. Cancer Res [Internet] 1992 [cited 2018 Mar 23];52(4):897-903. Available from: http://cancerres.aacrjournals.org/content/52/4/897.long.

43. Peters RH, Stuart RK. Synergism between 4-hydroperoxycyclophosphamide and cisplatin: importance of incubation sequence and measurement of cisplatin accumulation. Biochem Pharmacol [Internet]. 1990 [cited 2018 Mar 26];3(3):607-9. Available from: https://doi.org/10.1016/0006-2952(90)90070-2.

44. Waltmire CN, Alberts DS, Dorr RT. Sequence-dependent cytotoxicity of combination chemotherapy using paclitaxel, carboplatin and bleomycin in human lung and ovarian cancer. Anticancer Drugs. 2001;12(7):595-602.

45. Zamboni WC, Egorin MJ, Van Echo DA, Day RS, Meisenberg BR et al. Pharmacokinetic and pharmacodynamic study of the combination of docetaxel and topotecan in patients with solid tumors. J Clin Oncol [Internet]. 2000 [cited $2018 \mathrm{Mar}$

21];18(18):3288-94. Available from: https://doi.org/10.1200/JCO.2000.18.18.3288.

46. Torigoe S, Ogata Y, Matono K, Shirouzu K. Molecular Mechanisms of Sequence-dependent Antitumor Effects of SN-38 and 5Fluorouracil Combination Therapy against Colon Cancer Cells. Anticancer Res. 2009;29(6):2083-9.

47. Fischel JL, Rostagno P, Formento P, Dubreuil A, Etienne MC, Milano G. Ternary combination of irinotecan, fluorouracil-folinic acid and oxaliplatin: Results on human colon cancer cell lines. Br J Cancer. 2001;84(4):579-85.

48. Mans DR, Grivicich I, Peters GJ SG. Sequence-dependent growth inhibition and DNA damage formation by the irinotecan-5fluorouracil combination in human colon carcinoma cell lines. Eur J Cancer. 1999;35(13):1851-61.

49. Falcone A, Di Paolo A, Masi G, Allegrini G, Danesi R, Lencioni M et al. Sequence Effect of Irinotecan and Fluorouracil Treatment on Pharmacokinetics and Toxicity in Chemotherapy-Naive Metastatic Colorectal Cancer Patients. J Clin Oncol [Internet]. 2001 [cited 2018 Mar 19];19(15):3456-62. Available from: https://doi.org/10.1200/JC0.2001.19.15.3456.

50. Cattel L, Airoldi M, Passera R, Cagliero E, Stella B, Goffredo F. Gemcitabine plus vinorelbine chemotherapy regimens: A pharmacokinetic study of alternate administration sequences. Pharm World Sci. 2004;26(4):238-41.

51. Rowinsky EK, Gilbert MR, McGuire WP, Noe DA, Grochow LB, Forastiere AA et al. Sequences of topotecan and cisplatin: phase I, pharmacologic, and in vitro studies to examine sequence dependence. J Clin Oncol. 1991;9(9):1692-703.

52. Morelli MP, Cascone T, Troiani T, De Vita F, Orditura M, Laus G et al. Sequence-dependent antiproliferative effects of cytotoxic drugs and epidermal growth factor receptor inhibitors. Ann Oncol. 2005;16(Suppl. 4):6-10.

53. Tang Y, Wang Y, Teng X. Sequence-dependent effect of gemcitabine and cisplatin on A549 non-small-cell lung cancer cells. Mol Med Rep [Internet]. 2013 [cited 2018 Mar 10];8(1):221-6. Available from: https://doi.org/10.3892/mmr.2013.1495.

54. Voigt W, Bulankin A, Müller T, Schoeber C, Grothey A, Hoang-Vu C et al. Schedule-dependent antagonism of gemcitabine and cisplatin in human anaplastic thyroid cancer cell lines. Clin Cancer Res [Internet]. 2000 [cited 2018 Mar 20];6(5):2087-93. Available from: http://www.ncbi.nlm.nih.gov/pubmed/10815936.

55. Crinò L, Scagliotti G, Marangolo M, Figoli F, Clerici M, De Marinis F et al. Cisplatin-gemcitabine combination in advanced nonsmall-cell lung cancer: a phase II study. J Clin Oncol. 1997;15(1):297-303. 
56. Boss DS, Siegel-Lakhai WS, van Egmond-Schoemaker NE, Pluim D, Rosing H, Ten Bokkel Huinink WW et al. Phase I Pharmacokinetic and Pharmacodynamic Study of Carboplatin and Topotecan Administered Intravenously Every 28 Days to Patients with Malignant Solid Tumors. Clin Cancer Res [Internet]. 2009 [cited 2018 Mar 20];15(3):4775-83. Available from: https://doi.org/10.1158/1078-0432.CCR-08-3144.

57. Moore A. Evidence-based everything. Bandolier 1995;1(12).

58. Gil-Delgado MA, Bastian G, Guinet F, Spano JP, Taillibert S, Rocher MA et al. Oxaliplatin plus irinotecan and FU-FOL combination and pharmacokinetic analysis in advanced colorectal cancer patients. Am J Clin Oncol. 2004;27(3):294-8.

59. Airoldi M, Cattel L, Passera R, Pedani F, Delprino L, Micari C. Gemcitabine and oxaliplatin in patients with metastatic breast cancer resistant to or pretreated with both anthracyclines and taxanes: Clinical and pharmacokinetic data. Am J Clin Oncol Cancer Clin Trials. 2006;29(5):490-4.

60. Obasaju CK, Johnson SW, Rogatko A, Kilpatrick D, Brennan JM, Hamilton TC et al. Evaluation of carboplatin pharmacokinetics in the absence and presence of paclitaxel. Clin Cancer Res. 1996;2(3):549-52.

61. van Warmerdam L, Huizing MT, Giaccone G, Postmus PE, ten Bokkel Huinink WW, van Zandwijk N et al. Clinical pharmacology of carboplatin administered in combination with paclitaxel. Semin Oncol. 1997;24(1 Suppl 2):97-104.

62. Huizing MT, Giaccone G, van Warmerdam L, Rosing H, Bakker PJ, Vermorken JB et al. Pharmacokinetics of paclitaxel and carboplatin in a dose-escalating and dose-sequencing study in patients with non-small-cell lung cancer. J Clin Oncol.

1997;15(1):317-29.

63. Denlinger CS, Blanchard R, Xu L, Bernaards C, Litwin S, Spittle C et al. Pharmacokinetic analysis of irinotecan plus bevacizumab in patients with advanced solid tumors. Cancer Chemother Pharmacol. 2009;65(1):97-105.

64. Nakanishi Y, Takayama K, Wataya H, Izumi M, Minami T, Takano K et al. Phase I study of weekly irinotecan combined with weekly cisplatin in patients with advanced solid tumors. Chemotherapy [Internet]. 2002 [cited 2018 Mar 21];48(4):205-10.

Available from: http://www.ncbi.nlm.nih.gov/pubmed/12218268.

65. Gianni L, Munzone E, Capri G, Fulfaro F, Tarenzi E, Villani F et al. Paclitaxel by 3-hour infusion in combination with bolus doxorubicin in women with untreated metastatic breast cancer: high antitumor efficacy and cardiac effects in a dose-finding and sequence-finding study. J Clin Oncol. 1995;13(11):2688-99.

66. Bonassa EMA, Gato MIR. Terapêutica Oncológica para Enfermeiros e Farmacêuticos. 4 ed. São Paulo: Atheneu; 2012.

67. Pippa LF. Farmacocinética, metabolismo e excreção renal de doxorrubicina em pacientes com câncer de mama [dissertação]. Ribeirão Preto: USP; 2016.

68. Lal S, Sandanaraj E, Wong ZW, Ang PC, Wong NS, Lee EJ et al. CBR1 and CBR-3 pharmacogenetics and their influence on doxorubicin disposition in Asian breast cancer patients. Cancer Sci [Internet]. 2008 [cited 2018 Mar 26];99(10):2045-2054. Available from: https://doi.org/10.1111/j.1349-7006.2008.00903.x.

69. Sledge GW Jr, Robert N, Sparano JA, Cogleigh M, Goldstein L, Neuberg D et al. Eastern Cooperative Oncology Group Studies of Paclitaxel and Doxorubicin in Advanced Breast Cancer. Semin Oncol. 1995;22(3):105-8.

70. Cruz M, Duarte-Rodrigues J, Campelo M. Cardiotoxicidade na terapêutica com antraciclinas: estratégias de prevenção. Rev Port Cardiol [Internet]. 2016 [cited 2018 Mar 20];35(6):359-71. Available from: https://doi.org/10.1016/j.repc.2015.12.004.

71. Valdivieso M, Burgess MA, Ewer MS, Mackay B, Wallace S, Benjamin RS et al. Increased therapeutic index of weekly doxorubicin in the therapy of non-small cell lung cancer: a prospective, randomized study. J Clin Oncology.1984;2(3):207-14.

72. Legha SS, Benjamim RS, Mackay B, Ewer M, Wallace S, Valdivieso M et al. Reduction of doxorrubicin cardiotoxicity by prolonged continuous intravenous infusion. Ann Intem Med. 1982;96(2):133-9.

73. Ulas A, Silay K, Akinci S, Dede DS, Akinci MB, Sendur MAN et al. Medication errors in chemotherapy preparation and administration: A survey conducted among oncology nurses in Turkey. Asian Pacific J Cancer Prev [Internet]. 2015 [cited 2018 Mar 29];16(5):1699-705. Available from: http://journal.waocp.org/?sid=Entrez:PubMed\&id=pmid:25773812\&key=2015.16.5.1699.

74. Baldwin A, Rodriguez ES. Improving Patient Safety With Error Identification in Chemotherapy Orders by Verification Nurses. Clin J Oncol Nurs [Internet]. 2014 [cited 2018 Mar 21];20(1):59-65. Available from: https://doi.org/10.1188/16.CJON.59-65.

75. Boschi R, Rostagno E. Extravasation of antineoplastic agents: Prevention and treatments. Pediatr Rep [Internet]. 2012 [cited 2018 Mar 19];4(3):4-6. Available from: https://doi.org/10.4081/pr.2012.e28.

76. Wengström Y, Margulies A. European Oncology Nursing Society extravasation guidelines. Eur J Oncol Nurs [Internet]. 2008 [cited 2018 Mar 19];12(4):357-61. Available from: https://doi.org/10.1016/j.ejon.2008.07.003.

77. Hahn JC, Shafritz AB. Chemotherapy extravasation injuries. J Hand Surg Am [Internet]. 2012 [cited 2018 Mar 20];37(2):360-2. Available from: http://dx.doi.org/10.1016/j.jhsa.2011.10.028

78. Waters NJ. Evaluation of drug-drug interactions for oncology therapies: In vitro-in vivo extrapolation model-based risk assessment. Br J Clin Pharmacol [Internet]. 2015 [cited 2018 Mar 22];79(6):946-58. Available from:

http://dx.doi.org/10.1111/bcp.12563.

79. Fitzgerald KA, Malhotra M, Curtin CM, O'Brien FJ, O'Driscoll CM. Life in 3D is never flat: 3D models to optimise drug delivery. J Control Release [Internet]. 2015;215:39-54. Available from: http://dx.doi.org/10.1016/j.jconrel.2015.07.020.

80. Cruz DALM, Pimenta CAM. Prática baseada em evidências, aplicada ao raciocínio diagnóstico. Rev Latino-Am Enfermagem [Internet]. 2005 [cited 2018 Mar 29];13(3):415-22. Available from: http://dx.doi.org/10.1590/S0104-11692005000300017. 
81. NANDA Internacional. Diagnósticos de Enfermagem da NANDA-I: definições e classificação 2018-2020. 11a ed. Porto Alegre: Artmed; 2018.

82. Jomar RT, Gomes RAF, Leite DC, Gomes HF, Peres EM, Perez Junior EF. Nursing diagnoses in adult/elderly patients undergoing outpatient antineoplastic chemotherapy: a review. Ecancermedicalscience [Internet]. 2017 [cited 2018 Sept 01];11:736. Available from: https://doi.org/10.3332/ecancer.2017.736.

83. Gutiérrez MGR, Adami NP, Castro RAP, Fonseca SM. Natureza e classificação das intervenções de enfermagem em ambulatório de quimioterapia de adultos. Rev. Latino-Am. Enfermagem [Internet]. 2000 [cited 2018 Sept 01];8(3):33-9. Available from: http://dx.doi.org/10.1590/s0104-11692000000300006.

84. Alvim ALS. O processo de enfermagem e suas cinco etapas. Enferm Foco [Internet]. 2013 [cited 2018 Aug 31];4(2):140-1. Available from: https://doi.org/10.21675/2357-707X.2013.v4.n2.531.

85. Ferreira JN, Correia LRBR, Oliveira RM, Watanabe SN, Possari JF, Lima AFC. Managing febrile neutropenia in adult cancer patients: an integrative review of the literature. Rev Bras Enferm [Internet]. 2017 [cited 2018 Aug 31];70(6):1371-8. Available from: http://dx.doi.org/10.1590/0034-7167-2016-0247. 